\title{
Avaliação do rendimento de hidrodestilação para a produção de óleo essencial das cascas de Citrus sinensis (L.) Osbeck
}

\section{Evaluation of the hydrodistillation yield for the production of essential oil from peals of Citrus sinensis (L.) Osbeck}

\author{
Fúlvia Bezerra de Lima *1; Janaina Placedes *2; Cássia Regina Cardoso ${ }^{3}$ \\ ${ }^{*}$ Aluna do Curso de Graduação em Engenharia de Alimentos, Universidade Federal do Triângulo Mineiro, \\ Uberaba, Minas Gerais, Brasil. E-mail: fulvinha_bl@hotmail.com; ${ }^{2}$ janainaplacedes@hotmail.com \\ ${ }^{3}$ Professora do Curso de Engenharia de Alimentos, Universidade Federal do Triângulo Mineiro, Uberaba, \\ Minas Gerais, Brasil. E-mail: cassia.cardoso@uftm.edu.br
}

RESUMO: Resíduos da laranja são originados, em sua maior parte, da produção de suco de laranja. Esses resíduos podem ocasionar problemas ambientais e econômicos, devido ao processo de fermentação, ocasionado pela alta quantidade de água que possuem. Dentre as possíveis alternativas de aproveitamento dos resíduos da laranja está a obtenção de óleos essenciais. O presente trabalho objetivou analisar o rendimento do óleo essencial da casca de laranja através da hidrodestilação, variando a quantidade de água (solvente) e o tempo de extração. Os ensaios foram conduzidos baseados em um planejamento fatorial de dois níveis. Utilizou-se casca de laranja do tipo Pera, uma das mais produzidas no Brasil. Através do planejamento fatorial realizado no software Statistica, verificou-se que apenas a variável tempo foi determinante para o cálculo do rendimento. Assim, obteve-se uma equação preditiva para a estimativa de rendimento no período estudado. Experimentalmente, verificou-se que o rendimento mais alto foi obtido com a maior quantidade de solvente $(400 \mathrm{~mL}$ ) e maior tempo de extração (40 min); sendo este de aproximadamente $2,36 \%$ de óleo essencial. A densidade do óleo essencial foi determinada; $\rho=0,8356 \mathrm{~g} / \mathrm{mL}$; valor próximo aos encontrados na literatura.

Palavras-chave: Cascas de laranja; Planejamento fatorial; Extração de óleo.

ABSTRACT: Orange residues are mostly derived from the production of orange juice. These plant materials can cause environmental and economic problems, due to the fermentation process, caused by the high amount of water. Among the possible alternatives for the use of orange residues there is the production of essential oils. The objective of the present work was to analyze the yield of the essential oil of the orange peel through hydrodistillation, varying the amount of water (solvent) and the extraction time. The experiments were performed based on a two-level factorial design. The used peels were from Pera orange, one of the most produced varieties in Brazil. The factorial planning performed in the Statistica software, showed that that the variable time was determinant for the calculation of the yield. Thus, a predictive equation was obtained for the estimation of yield in the studied period. Experimentally, it was found that the highest yield was obtained with the highest amount of solvent $(400 \mathrm{~mL})$ and the longer extraction time (40 min); being approximately $2.36 \%$ of essential oil. The density of the essential oil was determined; $\rho=0.8356 \mathrm{~g} / \mathrm{mL}$; a value close to those found in the literature.

Keywords: Orange peels; Factorial planning; Oil extraction.

DOI: $10.18554 /$ rbcti.v4i3.3728 
INTRODUÇÃO

O Brasil é um dos maiores produtores mundiais de laranja (Citrus sinensis (L.) Osbeck), sendo sua maior parte destinada para produção de suco, o que acarreta alta geração de resíduos, despertando interesse pela utilização dos mesmos (LEÃO, 2015; ALEXANDRINO et al., 2007).

Consequentemente, há opções de reaproveitamento desses resíduos, dentre eles a maior parte é destinada para ração animal, porém existem outros possíveis usos como: obtenção de óleos essenciais, obtenção de compostos antioxidantes, fabricação de químicos, aplicação como fertilizantes orgânicos, produção de doce de casca de laranja cristalizada (REZZADORI; BENEDETTI, 2009).

Os óleos essenciais são compostos aromáticos voláteis que podem ser extraídos de diversas partes das plantas, como raízes, caules, folhas e flores (SERAGLIO et al., 2014). O estudo sobre plantas aromáticas é comum desde que as antigas civilizações usavam as plantas ou parte delas para vários propósitos, como cosméticos, para fins medicinais e religiosos. Atualmente, é crescente o interesse das indústrias por produtos que possuem compostos ativos naturais, para aplicações em cosméticos, alimentos, fármacos (LEÃO, 2015).

Há várias técnicas para extração de óleos essenciais, como hidrodestilação, destilação por arraste de vapor, prensagem a frio, utilização de solventes orgânicos e a extração com fluido supercrítico. A escolha do método depende da facilidade de evaporação e da hidrofobicidade do composto de interesse. Porém, a maior parte dos óleos essenciais é obtida pelo método de hidrodestilação, o qual utiliza vapor de água para arrastar o composto de interesse presente na planta (ENGINEERING IASTATE, 2019; KOKETSU; GONÇALVES, 1991).

O objetivo do presente trabalho foi obter óleo essencial a partir da casca da laranja do tipo Pera, uma das mais produzidas no Brasil, avaliando a influência do tempo de extração e da quantidade de solvente no rendimento de óleo gerado, empregando um planejamento experimental de dois fatores.

\section{MATERIAL E MÉTODOS}

Devido à geração de resíduo de laranja no Brasil, a biomassa utilizada para o presente projeto é a casca de laranja. Foi utilizada laranja do tipo Pera, em consequência do seu alto consumo no Brasil.

As laranjas Pera foram adquiridas no comércio de Uberaba/MG, sendo sempre obtidas em período próximo à extração.

\section{Extração do óleo essencial da laranja}

Para avaliar a evolução das quantidades de óleo extraídas com o tempo foram realizados ensaios utilizando $400 \mathrm{ml}$ de água e verificando a produção de óleo em diferentes tempos: $8,16,40,60,80$ e 120 min. A análise foi realizada em réplica. Para avaliar fatores como tempo de extração, quantidade de solvente e rendimento do óleo essencial da laranja, foi utilizado um planejamento fatorial com variações de tempo e de volume de água (solvente), como descrito na Tabela 1. Tais análises também foram realizadas em réplica. 
Tabela 1. Planejamento fatorial com variação no tempo de extração e volume de solvente.

\begin{tabular}{c|c|c}
\hline Ensaios & Tempo (min) & $\begin{array}{c}\text { Volume de água } \\
(\mathrm{mL})\end{array}$ \\
\hline 1 & 8 & 300 \\
\hline 2 & 40 & 300 \\
\hline 3 & 8 & 400 \\
\hline 4 & 40 & 400 \\
\hline
\end{tabular}

É usual o emprego de variáveis escalonadas ou codificadas em planejamentos de experimentos, no qual seus valores originais são transformados em -1 e +1 , sendo esses, os níveis inferior e superior, respectivamente. $O$ escalonamento é apresentado através das Equações 1 e 2:

$$
\begin{gathered}
x_{i}^{-}=\frac{X_{i}^{-}-\bar{X}_{i}}{\frac{X_{i}^{+}-X_{i}^{-}}{2}}=-1 \\
x_{i}^{+}=\frac{X_{i}^{+}-\bar{X}_{i}}{\frac{X_{i}^{+}-X_{i}^{-}}{2}}=+1
\end{gathered}
$$

em que $\bar{X}_{i}$ representa o valor médio original entre os níveis inferior e superior do fator $i$, já $\mathrm{X}_{\mathrm{i}}^{-} \mathrm{e} \mathrm{X}_{\mathrm{i}}^{+}$, representam os valores originais do fator $\mathrm{i}$, nos níveis inferior e superior (CALADO; MONTGOMERY, 2003).

Portanto, escalonou-se a variável tempo $\left(\mathrm{X}_{1}\right)$ e obteve-se para o tempo de 8 e 40 min, respectivamente, através das Equações 3 e 4:

$$
\begin{gathered}
X_{1}^{-}=\frac{8-24}{\frac{40-8}{2}}=-1 \\
X_{1}^{+}=\frac{40-24}{\frac{40-8}{2}}=+1
\end{gathered}
$$

Escalonou-se também a variável volume $\left(\mathrm{X}_{2}\right)$ para 300 e $400 \mathrm{~mL}$, respectivamente:

$$
\begin{aligned}
& X_{2}^{-}=\frac{300-350}{\frac{400-300}{2}}=-1 \\
& X_{2}^{+}=\frac{\frac{400-350}{\frac{400-300}{2}}=+1}{}
\end{aligned}
$$

Para a extração do óleo essencial foi utilizada a casca de laranja, removida do fruto 
com o auxílio de um descascador, de modo a separar apenas o flavedo, casca exterior, onde está presente o óleo essencial.

Para cada análise, foram utilizados $100 \mathrm{~g}$ de casca de laranja (Figura 1a), o tempo de extração foi variado em 8 e 40 min e a quantidade de solvente variou em 300 e 400 $\mathrm{mL}$. A justificativa do uso desses intervalos de tempo se dá pelo fato da extração do óleo essencial ocorrer predominantemente no início do processo, após a ebulição da mistura. O gráfico ilustrando esses dados será apresentado na seção de resultados. Após atingir o ponto de ebulição iniciou-se a contagem dos tempos apresentados na Tabela 1. Adicionou-se água através do funil de separação durante a destilação, para manter o nível original da água no balão (Figura 1b).

Figura 1. Cascas de laranja (a) e nível inicial de água marcado no balão (b).

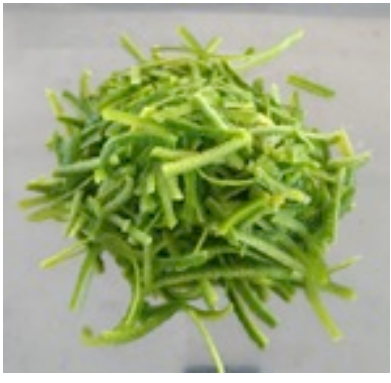

(a)

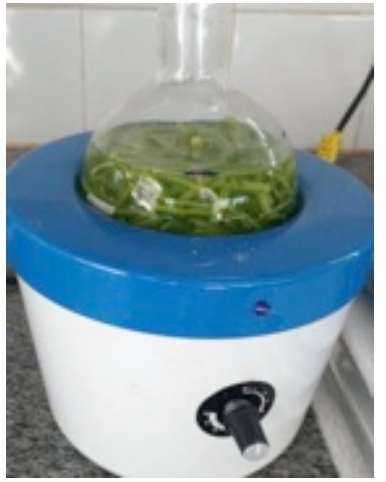

(b)

Após a adição de $100 \mathrm{~g}$ de cascas de laranja no balão de destilação de $500 \mathrm{~mL}$, adicionou-se o volume desejado de água. É conveniente o uso da biomassa (fonte de óleo) submersa em água para que a temperatura da amostra seja mantida constante, não ultrapassando $100^{\circ} \mathrm{C}$ e reduzindo o efeito de degradação de compostos voláteis (LOPES, 2014).

Foi realizado o aquecimento através de uma manta aquecedora até que a mistura entrasse em ebulição, liberando o vapor que arrastou o óleo presente na casca da laranja. Esse óleo e o vapor de água foram direcionados ao condensador, a mistura foi resfriada e retornou à fase líquida. O esquema utilizado para a extração do óleo está apresentado na Figura 2.

O óleo e a água, provenientes da condensação do vapor, foram coletados em um funil de separação no fim do condensador, sendo que o funil continha $0,5 \mathrm{~g}$ de $\mathrm{NaCl}$ e 10 $\mathrm{mL}$ de água para facilitar a separação das fases oleosa e aquosa, promovendo o efeito salting out.

A redução da fração aquosa na fase oleosa em decorrência de um considerável aumento de concentração iônica do meio, geralmente causado pela ação de um sal inorgânico, é chamada de efeito salting out. Se a uma mistura de água e óleo for adicionada uma determinada quantidade de sal, a fase aquosa apresentará um alto poder de solvatação dos íons, assim, a dissolução de água no óleo é reduzida uma vez que as moléculas de água interagem preferencialmente com os íons, forçando a separação da mistura de óleo e água (Faculdade de Ciências Farmacêuticas - UNESP, 2019). 
Figura 2. Sistema utilizado para extração de óleo essencial de laranja por hidrodestilação.

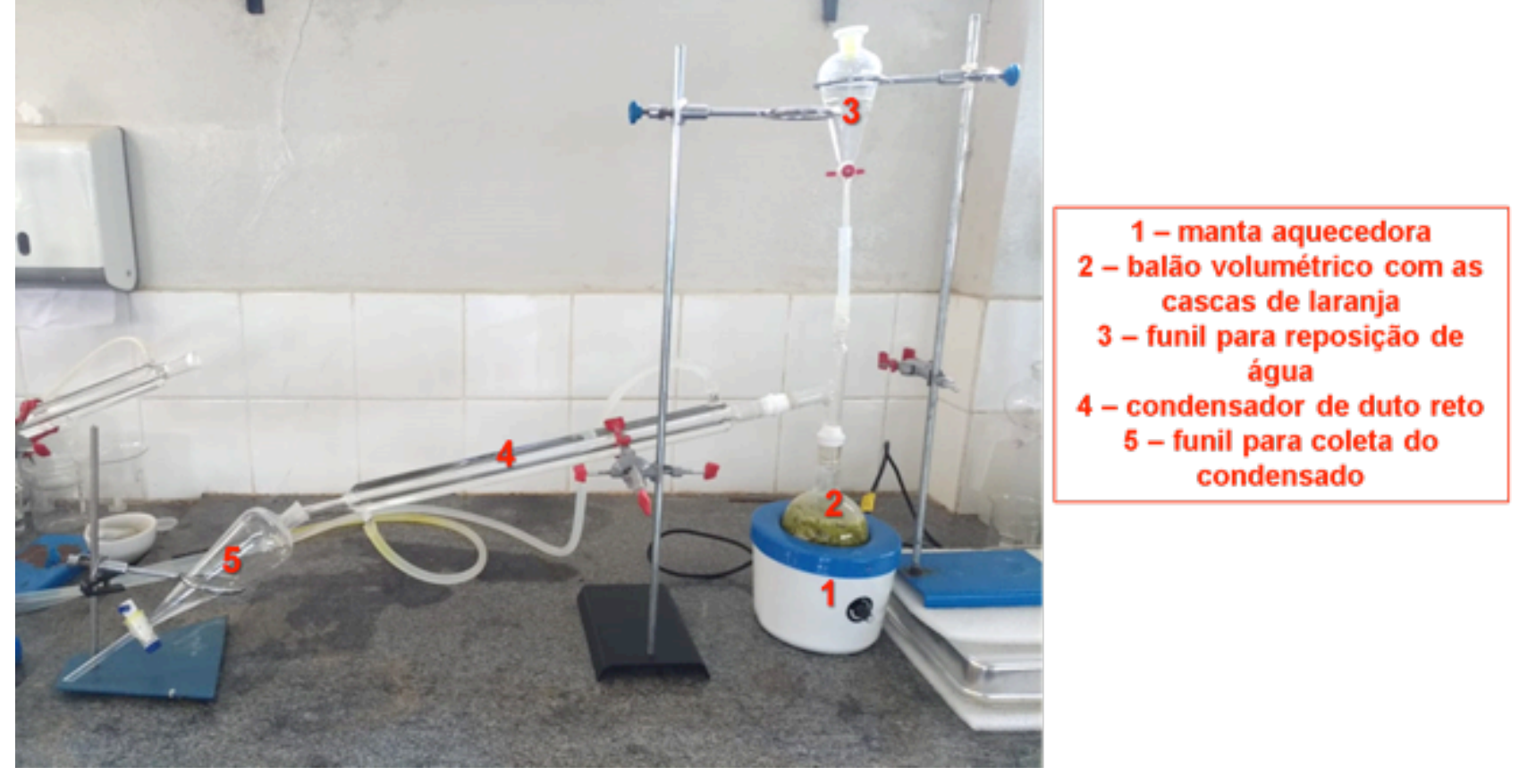

Após cada intervalo de tempo, foi necessária a troca do funil de separação por outro contendo a mesma solução de $\mathrm{NaCl}$ e água, para o próximo intervalo de tempo. Após a remoção do funil, adicionou-se a matriz de cor (óleo essencial da laranja e corante dissolvido), aproximadamente $0,5 \mathrm{~mL}$. Tal procedimento foi adotado buscando facilitar a visualização da separação de fases.

Para cada funil que era retirado do sistema, esperou-se cerca de $5 \mathrm{~min}$ a fim de garantir a separação de fases. Posteriormente, a fase aquosa foi descartada, a mesma se encontrava na parte inferior do funil. A fase oleosa foi transferida para um tubo de ensaio.

Deixou-se o tubo de ensaio em repouso por cerca de 5 minutos, para ocorrer a decantação, em seguida, coletou-se o óleo presente na superfície do tubo de ensaio utilizando micropipeta. Após tal procedimento, a fase oleosa foi transferida para um frasco âmbar, a fim de evitar a degradação da mesma por ação da luz ambiente, em seguida realizou-se a pesagem a fim de se calcular o rendimento.

$O$ rendimento experimental $\left(\eta_{\exp }\right)$ de óleo essencial foi calculado segundo a Equação 7 , sendo $\mathrm{m}_{\text {oleo }}$ a massa de óleo obtida em determinado tempo de extração e $\mathrm{m}_{\text {cascas }}$ a massa de cascas de laranja utilizada em cada análise.

$$
\eta_{\text {exp }}=\left(\frac{m_{\text {oleo }}}{m_{\text {cascas }}}\right) \cdot 100 \%
$$

\section{Determinação da densidade do óleo essencial da laranja.}

Para o cálculo da densidade do óleo essencial obtido, um balão volumétrico de 5 $\mathrm{mL}$ foi calibrado com água destilada. Após, o balão foi preenchido com óleo essencial de laranja até o menisco, em réplica; e a massa foi medida em balança analítica.

Sabe-se que o balão volumétrico não é a vidraria mais adequada para medição de densidade de líquidos, porém, o balão de volume $5 \mathrm{~mL}$ era a vidraria disponível para a medida considerando o pequeno volume de óleo essencial obtido nas extrações. 


\section{RESULTADOS E DISCUSSÃO}

\section{Extração do óleo essencial de laranja}

Considerando os procedimentos mencionados para a realização da extração do óleo essencial da casca de laranja através de hidrodestilação, foi possível avaliar a influência de fatores como tempo de extração e quantidade de solvente no rendimento do óleo essencial desejado.

Para volumes de solvente com $400 \mathrm{~mL}$ de água, observou-se a variação de rendimento de óleo em função do tempo, como mostra a Figura 3. Desse modo, os níveis de tempo escolhidos para o planejamento fatorial foram 8 e $40 \mathrm{~min}$. A 40 min observou-se em torno de $80 \%$ da extração total. Desse modo, a equação matemática a ser obtida no planejamento fatorial representaria o período de extração mais significativo.

Figura 3. Extração com $400 \mathrm{~mL}$ de água variando tempo de 0 a 120 min versus rendimento experimental de óleo essencial.

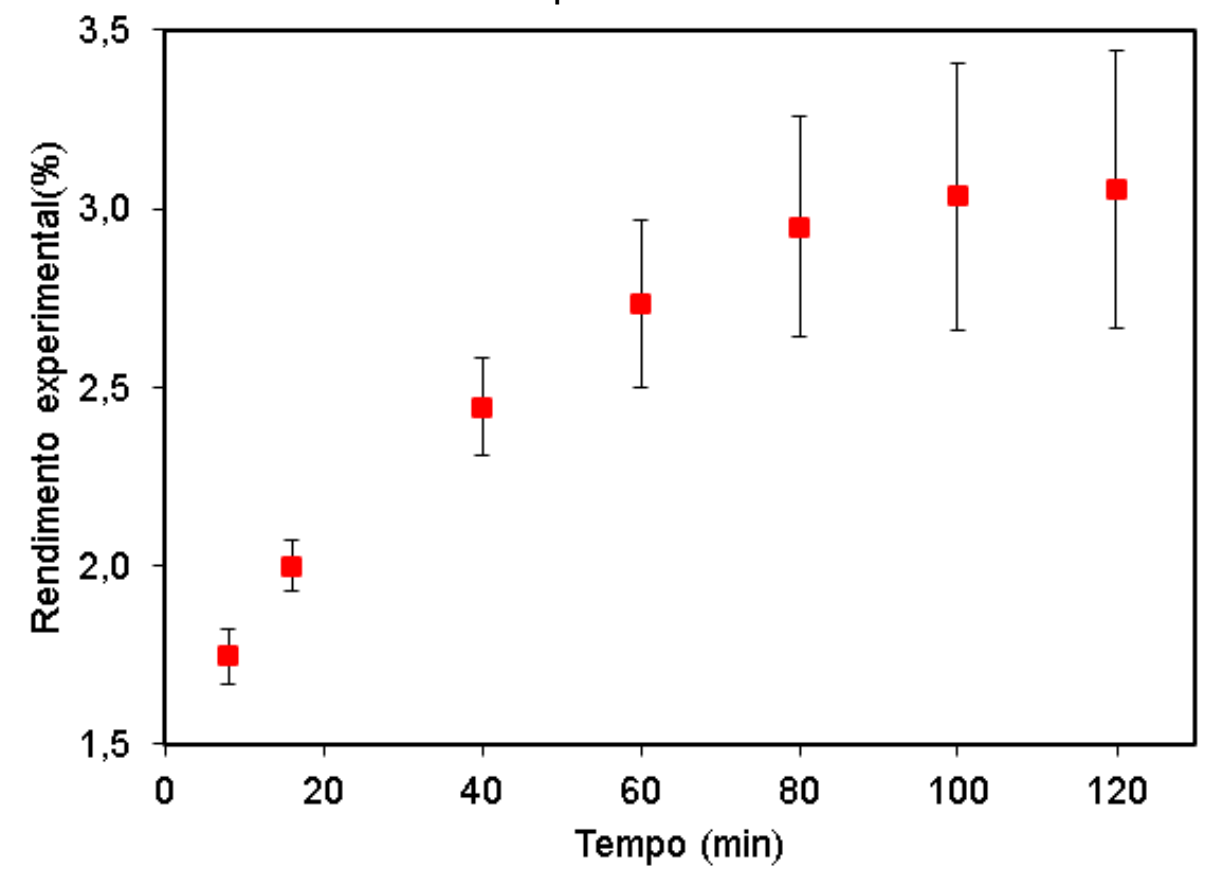

Na Tabela 2, encontra-se o planejamento experimental com os seus respectivos rendimentos.

Através do software Statistica versão 7.0, os dados experimentais foram tratados. Os parâmetros significativos para a estimativa de rendimento da extração do óleo essencial da laranja são apresentados na Tabela 3.

Sabe-se que para se avaliar a influência das variáveis estudadas e da interação entre elas na predição da variável dependente do modelo, observa-se o valor do parâmetro $\mathrm{p}$ (probabilidade de significância), que representa a probabilidade de erro envolvida em rejeitar a ideia de que a relação em questão não é significativa ou não modifica o valor da variável dependente. $O$ intervalo de confiança adotado na análise foi de $95 \%$; valores do parâmetro $p$ inferiores a 0,05 são considerados referentes às variáveis ou interações significativas para o cálculo da variável dependente (CALADO; MONTGOMERY, 2003). 
Tabela 2. Planejamento experimental com rendimentos obtidos em duplicata.

\begin{tabular}{c|c|c|c|c}
\hline Ensaios & 1 & 2 & 3 & 4 \\
\hline $\mathrm{X}_{1}$ & -1 & +1 & -1 & +1 \\
\hline $\mathrm{X}_{2}$ & -1 & -1 & +1 & +1 \\
\hline Tempo (min) & 8 & 40 & 8 & 40 \\
\hline Volume solvente (mL) & 300 & 300 & 400 & 400 \\
\hline Rendimento 1 (\%) & 1,8022 & 2,3144 & 1,8022 & 2,2944 \\
\hline Rendimento 2 (\%) & 1,9522 & 2,2744 & 1,9622 & 2,3644 \\
\hline Média (\%) & 1,8772 & 2,2944 & 1,7472 & 2,3294 \\
\hline
\end{tabular}

Tabela 3. Coeficientes de regressão, p-valores e efeitos para o planejamento fatorial $2^{2}$.

\begin{tabular}{c|c|c|c}
\hline Fatores & Efeitos & $p-$ valor & Coeficientes de regressão \\
\hline Média & 2,0958 & 0,0000 & 2,0958 \\
\hline 1 (tempo) & 0,4322 & 0,0018 & 0,2161 \\
\hline 2 (volume) & 0,0200 & 0,7493 & 0,0100 \\
\hline Interação (1,2) & 0,0150 & 0,8100 & 0,0075 \\
\hline
\end{tabular}

Logo, a Tabela 3 mostra que as variáveis volume e a interação "volume $x$ tempo" não foram significativas para a predição do rendimento da extração, já que os valores do parâmetro p são superiores a 0,05. Já o tempo de extração se mostrou significativo para a predição do rendimento $(p<0,05)$.

$\mathrm{Na}$ Tabela 4, encontram-se os coeficientes de regressão, p-valores e os efeitos que são significativos.

Tabela 4. Coeficientes de regressão, p-valores e os efeitos para o planejamento fatorial $2^{2}$, para os efeitos significativos.

\begin{tabular}{c|c|c|c}
\hline Fatores & Efeitos & $p-$ valor & Coeficientes de regressão \\
\hline Média & 2,0958 & 0,0000 & 2,0958 \\
\hline 1 (tempo) & 0,4322 & 0,0001 & 0,2161 \\
\hline
\end{tabular}

Com base nos resultados, obteve-se o modelo codificado de acordo com a Equação 8, sendo $\eta_{\text {calc }} \circ$ rendimento calculado de óleo, $t(x) \circ$ tempo de extração escalonado.

$$
\eta_{\text {calc }}(\%)=2,0958+0,2161 t(x)
$$

O coeficiente de determinação para a Equação 8 é $R^{2}=0,929$; sendo $t(x)$ calculado em relação ao tempo de extração em minutos, como mostra a Equação 9:

$$
t(x)=0,0625(x)-1,5
$$

em que $x$ representa o tempo de extração em minutos.

$\mathrm{Na}$ Tabela 5 encontra-se a análise de variância (ANOVA), para o modelo representado pela Equação (8). 
Tabela 5. Análise de variância (ANOVA) para o modelo representado pela Equação 8.

\begin{tabular}{c|c|c|c|c}
\hline Fatores & $\mathrm{SQ}$ & $\mathrm{GL}$ & $\mathrm{MQ}$ & $\mathrm{F}_{\text {cal }}$ \\
\hline 1 (tempo) & 0,3736 & 1 & 0,3736 & 78,51 \\
\hline Erro & 0,0286 & 6 & 0,0048 & - \\
\hline Total & 0,4021 & 7 & - & - \\
\hline
\end{tabular}

$\mathrm{SQ}, \mathrm{GL}$ e MQ representam, respectivamente, a soma dos quadrados, graus de liberdade e média dos quadrados.

Para avaliar a representatividade e a previsibilidade do modelo foram avaliados os valores do teste $F$, provenientes da Distribuição de Fisher-Snedecor. Para o modelo ser representativo e preditivo para o intervalo de dados experimentais estudados, o valor de $\mathrm{F}$ calculado $\left(F_{\text {calc }}\right)$, referente ao modelo ajustado, deve ser cerca de dez vezes maior que o valor tabelado $\left(F_{\text {tab }}\right)$, na distribuição referente ao nível de confiança adotado (CALADO; MONTGOMERY, 2003). O valor de $F_{\text {calc }}$ foi 78,51 e o valor de $F_{\text {tab }}(F 1,6 ; 95 \%)$ é 5,99 . Logo, o valor de $\mathrm{F}_{\text {calc }}$ é treze vezes maior que o tabelado. Assim, conclui-se que a equação além de ser representativa, é preditiva para o intervalo de tempo de 8 a 40 min.

A Figura 4 apresenta a superfície de resposta para o rendimento de óleo essencial relacionado com o tempo de extração e volume de solvente.

Figura 4. Superfície de resposta para o rendimento de óleo essencial relacionando com o tempo de extração e volume de solvente (variáveis escalonadas).

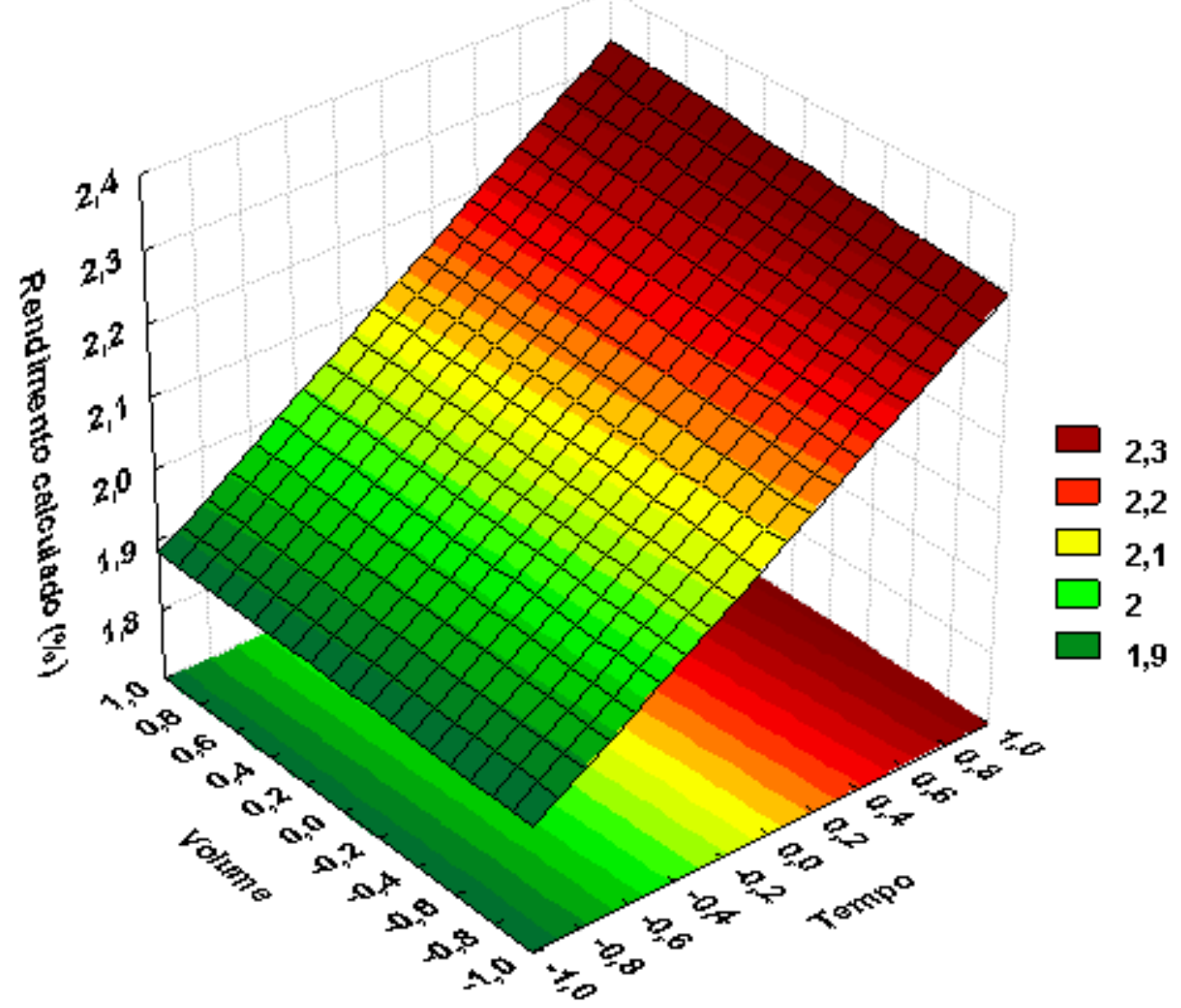


Figura 5. Valores normais esperados versus resíduos.

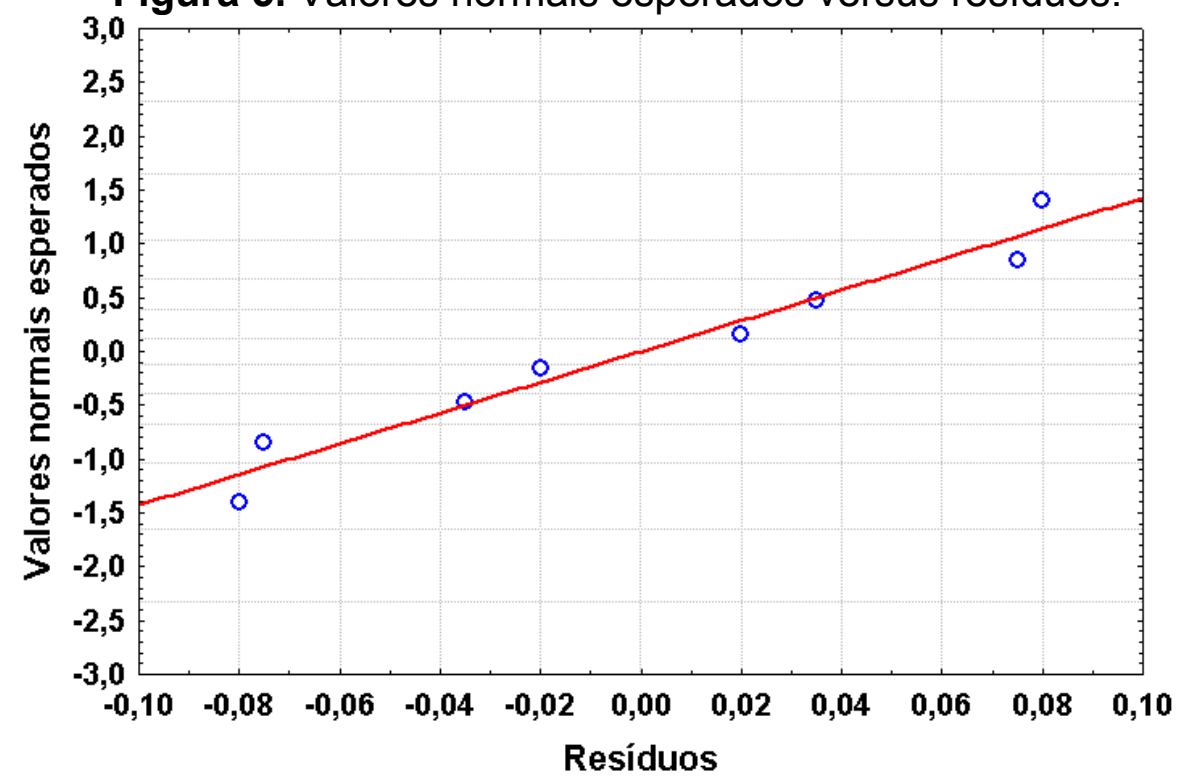

Observa-se na Figure 4 que o rendimento varia com o tempo de extração, e não com o volume, na faixa experimental adotada. Na Figura $\mathbf{5}$ encontra-se os valores normais esperados versus os resíduos. Quanto mais próximos os pontos que representam os resíduos estiverem da linha contínua, mais válida será a suposição de normalidade para os resíduos.

\section{Determinação da densidade do óleo essencial de laranja}

As medidas de densidade para óleo essência de laranja resultaram em valor de $\rho=$ $0,8356 \pm 0,0012 \mathrm{~g} / \mathrm{mL}$.

Como mencionado anteriormente, o principal constituinte do óleo de casca de laranja é o limoneno. O valor de densidade para o limoneno a $20^{\circ} \mathrm{C}$ é $0,842 \mathrm{~g} / \mathrm{mL}$ (SIGMA - ALDRICH, 2019). Já a densidade do óleo essencial de laranja varia de 0,840 - 0,850 $\mathrm{g} / \mathrm{mL}$ (HILDEBRAND ALIMENTOS, 2019). Portanto, a densidade obtida no presente trabalho apresentou valores próximos aos valores encontrados na literatura, indicando que os processos de extração de óleo e separação de fases foram realizados de maneira eficiente.

\section{CONCLUSÕES}

A influência das variáveis tempo de extração e volume de solvente foi avaliada para a hidrodestilação da casca de laranja Pera.

O maior rendimento obtido foi de $2,3644 \%$ para tempo de 40 min e volume de solvente de $400 \mathrm{~mL}$.

A variável volume e a interação "volume $x$ tempo" não foram significativas para a determinação do rendimento de óleo, já o tempo de extração foi significativo para a predição de rendimentos.

O modelo além de ser representativo, é preditivo para os intervalos de dados experimentais estudados.

A densidade medida para o óleo obtido no presente trabalho foi determinada e 
apresentou valores similares aos presentes na literatura para o limoneno e para óleos essenciais de laranja, indicando que os processos de extração de óleo e separação de fases foram realizados de maneira eficiente.

\section{REFERÊNCIAS}

ALEXANDRINO, A.M. et al. Aproveitamento do resíduo de laranja para a produção de enzimas lignocelulolíticas por Pleurotus ostreatus (Jack:Fr). Ciência e Tecnologia de Alimentos, v. 27, n. 2, p. 364-368, 2007.

CALADO, V.; MONTGOMERY, D. Planejamento de Experimentos usando o

Statistica: volume 1. 1 ed. Rio de Janeiro: E-papers Serviços Editoriais, 2003.

ENGINEERING IASTATE. Essential Oils from Steam Distillation. Disponível em: http://www.engineering.iastate.edu/brl/files/2011/10/brl_essentialoils.pdf. Acesso em: 10 maio 2019.

FACULDADE DE CIÊNCIAS FARMACÊUTICAS - UNESP. Propriedades gerais das proteínas. Disponível em:

http://www.fcfar.unesp.br/alimentos/bioquimica/praticas_proteinas/precipitacao_proteinas. htm. Acesso em: 11 maio 2019.

HILDEBRAND ALIMENTOS. Óleos Essenciais. Disponível em:

http://www.hildebrand.com.br/oleos\%20essenciais.php. Acesso em: 04 abril 2018.

KOKETSU, M.; GONÇALVES, S.L. Óleos essenciais e sua extração por arraste a vapor. Disponível em: https://www.agencia.cnptia.embrapa.br/Repositorio/doc81991_000gc3p3vcp02wx5ok01dx9lc4wnplbh.pdf. Acesso em: 02 jul. 2019.

LEÃO, M. Análise do óleo essencial da laranja doce Citrus sinensis (L.) Osbeck obetido das cascas secas e frescas através do método de extração por

hidrodestilação. TCC (Graduação em Farmácia) - Universidade de Santa Cruz do Sul, Santa Cruz do Sul, 2015.

LOPES, J.F.D. Cultivo e processamento de plantas aromáticas. Dissertação.

(Mestrado em Tecnologia e Segurança Alimentar) - Faculdade de Ciências e Tecnologia Universidade Nova de Lisboa, Lisboa, 2014.

REZZADORI, K.; BENEDETTI, S. Proposições para valorização de resíduos do processamento do Suco de Laranja. In: International Workshop advances in cleaner production. Florianópolis, 2009.

SERAGLIO, J. et al. Extração de óleo essencial da casca da canela sassafrás. In: XX Congresso Brasileiro de Engenharia Química. Florianópolis, 2014.

SIGMA - ALDRICH. Disponível em:

https://www.sigmaaldrich.com/catalog/product/sigma/183164?lang=pt\&region=BR.

Acesso em: 19 abril. 2019.

Recebido em: 13/05/2019

Aprovado em: 02/07/2019 